\title{
Plantas hospedeiras e galhas entomógenas em sub-bosques de florestas tropicais do Pará, Brasil
}

\author{
Walter Santos de Araújo ${ }^{1}$; Éder Dasdoriano Porfírio-Júnior ${ }^{2}$; \\ Vanessa Araújo Jorge ${ }^{3}$ \& Kleber do Espírito-Santo Filho ${ }^{4}$
}

Enviado em maio de 2012; aceito em novembro de 2012.

\section{Resumo}

Nesse estudo nós registramos a ocorrência de galhas e plantas hospedeiras em áreas de sub-bosque de Floresta Ombrófila Densa, na Floresta Nacional Saracá-Taquera em Porto Trombetas, norte do Pará, Brasil. Foram registrados 112 morfotipos de galhas distribuídos em 65 espécies e 33 famílias de plantas hospedeiras, sendo Cecidomyiidae (Diptera) o grupo de galhadores mais importante. As famílias de plantas que apresentaram maior riqueza de galhas foram Fabaceae com 18 morfotipos, Bignoniaceae com 14, Lauraceae e Sapotaceae com 12, e Burseraceae com 10 espécies de insetos galhadores. Os gêneros Adenocalymma (Bignoniaceae), Pouteria (Sapotaceae), Inga (Fabaceae) e Protium (Burseraceae) e as espécies Adenocalymma neoflavidum(Bignoniaceae) e Ocotea sp. (Lauraceae) foram os táxons mais diversos de plantas hospedeiras. A riqueza de galhas na floresta estudada é elevada quando comparada a outros biomas brasileiros, como o Cerrado e a Mata Atlântica. Esses resultados mostram a importância da região amazônica para a diversidade de galhas entomógenas no Brasil.

Palavras-chave: Amazônia, Fabaceae, insetos galhadores, plantas hospedeiras.

DOI: http://dx.doi.org/10.5007/2178-4574.2012n41p59

1. Laboratório de Entomologia, Departamento de Ecologia, Instituto de Ciências Biológicas, Universidade Federal de Goiás, Campus II, Caixa Postal 131, 74001-970, Goiânia, GO, Brasil. E-mail:

walterbioaraujo@yahoo.com.br

2. Laboratório de Morfologia e Taxonomia Vegetal, Departamento de Biologia Geral, Instituto de Ciências

Biológicas, Universidade Federal de Goiás, Campus II, Caixa Postal 131, 74001-970, Goiânia, GO,

Brasil.

3. Laboratório de Análises e Gerenciamento Ambiental de Recursos Hídricos. Universidade Federal de

Goiás, Campus II, Caixa Postal 131, 74001-970, Goiânia, GO, Brasil.

4. Faculdade de Engenharia Ambiental, Universidade Federal de Goiás, Campus II, Caixa Postal 131,

74001-970, Goiânia, GO, Brasil.

Financiado pela Mineração Rio Norte - MRN.

\section{(cc) BY-NE}

Este artigo é de Acesso Livre, disponibilizado sob os termos da

Creative Commons Attribution 3.0 Unported License (http://creativecommons.org/licenses/by/3.0/) que permite uso não-comercial, distribuição e reprodução em qualquer meio, desde que este trabalho original seja devidamente citado. 


\section{Abstract}

(Host plants and entomogenous galls in understory of tropical forests in the Pará, Brazil) In this study we recorded the occurrence of galls and host plants in understory areas of Floresta Ombrófila Densa, in the Floresta Nacional de SaracáTaquera in Porto Trombetas, north of Pará, Brazil. We recorded 112 gall morphotypes distributed in 65 families and 33 species of host plants, being Cecidomyiidae (Diptera) the most important group of galling insects. The plant families that showed more gall richness were Fabaceae with 18 morphotypes, Bignoniaceae with 14, Lauraceae and Sapotaceae with 12, and Burseraceae with 10 galling species. The genera Adenocalymma (Bignoniaceae), Pouteria (Sapotaceae), Inga (Fabaceae) and Protium (Burseraceae) and the species Adenocalymma neoflavidum (Bignoniaceae) and Ocotea sp. (Lauraceae) were the most diverse host plant taxa. Gall richness in the studied forest is high when compared to others Brazilian biomes, as Cerrado and Mata Atlântica. These results show the importance of Amazon region to diversity of entomogenous galls in the Brazil.

Key words: Amazon, Fabaceae, galling insects, host plants.

\section{Introdução}

As florestas tropicais são conhecidas por abrigar a maior diversidade de insetos herbívoros do mundo (Godfray et al. 1999). Essa alta diversidade deve-se, principalmente ao elevado nível de estratificação e especialização dos grupos de herbívoros, associada à alta diversidade de plantas hospedeiras (Lewinsohn et al. 2005). Segundo Godfray et al. (1999), conhecer e entender essa grande diversidade são os principais desafios para os biólogos e ecólogos modernos. Nesse contexto, a Amazônia brasileira destaca-se como importante região para estudos e inventários sobre a diversidade de insetos herbívoros.

Insetos galhadores são uma guilda de herbívoros que modificam os tecidos vegetais por hipertrofia e hiperplasia das células, induzindo a formação de galhas (Stone e Schönrogge 2003). A maioria das espécies conhecidas é especialista ocorrendo em apenas uma única espécie de planta hospedeira (Carneiro et al. 2009a). No Brasil, historicamente os estudos de insetos galhadores foram mais concentrados no Cerrado (Maia \& Fernandes 2004; Coelho et al. 2009b; Santos et al. 2010; Araújo et al. 2011) e na Mata Atlântica (Maia 2001; Maia et al. 2008; Fernandes et al. 2009). Entretanto, recentemente alguns estudos desenvolvidos na Amazônia brasileira tem mostrado que a região é uma das mais ricas do Brasil em galhas de insetos e.g. (Julião et al. 2005; Almada \& Fernandes 2011).

O presente trabalho foi desenvolvido na porção Oriental da Amazônia brasileira em áreas de sub-bosque de floresta nativa na Floresta Nacional de SaracáTaquera, em Porto Trombetas, Pará, Brasil. Recentemente, Almada \& Fernandes (2011) registraram 309 espécies de galhadores na região, entretanto, as coletas foram 
focadas em áreas de reflorestamento (vegetação secundária). Em outro estudo, Maia (2011) publicou a listagem de insetos de uma área de platô da região (Platô Bacaba) registrando 76 morfotipos de galhas de insetos. Assim, o objetivo desse estudo foi realizar um levantamento de galhas de insetos e suas plantas hospedeiras em áreas de sub-bosque de Floresta Ombrófila Densa na Floresta Nacional de Saracá-Taquera, bem como comparar os resultados obtidos com outros trabalhos da região e do bioma Amazônia.

\section{Material e Métodos Área de Estudo}

O estudo foi conduzido nas áreas de vegetação remanescentes de extração de bauxita da Mineração Rio do Norte na Floresta Nacional Saracá-Taquera em Porto Trombetas, município de Oriximiná, estado do Pará, Brasil ( $\left.1^{\circ} 38^{\prime} \mathrm{S}, 56^{\circ} 26^{\prime} \mathrm{W}\right)$. As áreas estudadas localizam-se aproximadamente a $65 \mathrm{~km}$ a nordeste da cidade de Oriximiná e a 30km do Rio Trombetas, apresentando uma altitude média 180 metros acima do nível do mar. O clima da região apresenta estações chuvosa (inverno) e seca (verão) distintas, com média mensal de precipitação excedendo os $10 \mathrm{~mm}$ na maioria dos meses do ano. A temperatura média máxima e mínima de temperatura é de $34,6^{\circ}$ e $19,9^{\circ} \mathrm{C}$, respectivamente (Fernandes et al. 2010).

A Floresta Nacional de Saracá-Taquera está localizada na área de Domínio das Terras Baixas Florestadas da Amazônia, sendo a Floresta Ombrófila Densa a vegetação predominante na região (IBGE 1992). Essa formação caracteriza-se pela grande biomassa vegetal, pela existência de um dossel uniforme onde predominam as Pouteria spp. (Sapotaceae) e Protium spp. (Burseraceae) e pela presença de árvores emergentes com até $45 \mathrm{~m}$ de altura, onde se destaca Dinizia excelsa (Fabaceae).

Nas áreas de extração de bauxita da região, a floresta nativa está localizada na borda de platôs. Os platôs são áreas de relevo elevado, que formam pequenos morros, onde há grande concentração de minério. Como a extração do minério se dá no topo dos platôs, há supressão da vegetação nativa nessa porção, restando apenas floresta nas encostas. No inventário, foram amostrados os seguintes platôs: Almeidas, Aviso, Bacaba, Bela Cruz, Monte Branco, Papagaio, Periquito e Saracá.

\section{Amostragem}

A amostragem de galhas foi realizada em duas campanhas de aproximadamente 20 dias cada, sendo uma no período seco (setembro de 2010) e outra no período chuvoso (março de 2011). A amostragem foi feita através de 22 transectos estabelecidos perpendicularmente às bordas dos platôs em um desenho amostral do tipo "espinha de peixe". Cada transecto apresentava $500 \mathrm{~m}$ de comprimento e continha quatro linhas perpendiculares de $200 \mathrm{~m}$ de largura por $2 \mathrm{~m}$ de comprimento, onde foram feitas as coletas de galhas. Nos platôs Almeidas, Aviso, Papagaio, Periquito e Saracá foram amostrados dois transectos, já nos platôs Bacaba, 
Bela Cruz e Monte Branco foram amostrados quatro transectos. Todas as linhas foram percorridas por dois coletores (sempre os mesmos) que vistoriaram todas as plantas até uma altura de $2,5 \mathrm{~m}$ em busca de galhas.

Características morfológicas das galhas foram registradas em campo, tais como, órgão de ocorrência, forma, coloração, pilosidade e tamanho. Além disso, fragmentos das plantas hospedeiras foram coletados e herborizados para posterior identificação. As plantas foram identificadas por consulta a especialistas, bem como comparação com literatura especializada e com as coleções dos herbários da Universidade Federal de Goiás e do Instituto de Pesquisas da Amazônia. Os autores das espécies, nomenclaturas e sinonímias foram consultados no banco de dados The Plant List (2010).

Os morfotipos de galhas foram utilizados como indicadores das espécies de insetos galhadores, uma vez que são únicos para cada espécie de inseto (Carneiro et al. 2009a). Os insetos adultos não foram obtidos devido ao curto tempo para realização do trabalho, sendo que em alguns casos as galhas foram dissecadas em campo para determinação do grupo galhador. Além disso, praticamente todas as espécies de insetos galhadores da Amazônia são desconhecidas tornando sua identificação taxonômica bastante difícil (Fernandes et al. 2010). Diversos estudos têm utilizado morfotipos de galhas como indicadores das espécies de insetos galhadores (ver Carneiro et al. 2009a).

\section{Resultados e Discussão}

Foram registrados 112 morfotipos de galhas distribuídos em 65 espécies e 33 famílias de plantas hospedeiras (Tabela 1). Para a maioria dos morfotipos não foram determinados os táxons dos galhadores, entretanto, todos os insetos pertenciam à família Cecidomyiidae (Diptera). As famílias de plantas que apresentaram maior riqueza de galhas foram Fabaceae com 18 morfotipos, Bignoniaceae com 14, Lauraceae e Sapotaceae com 12, e Burseraceae com 10 morfotipos (Tabela 2).

Estudos anteriores na Amazônia também registraram Fabaceae como a família de plantas hospedeiras mais diversa em número de espécies de galhadores (Julião et al. 2005; Almada \& Fernandes 2011). Resultados semelhantes também têm sido encontrados para áreas do Cerrado brasileiro (ver Maia e Fernandes 2004; Santos et al. 2010; Araújo et al. 2011). Esse padrão pode ser explicado pela alta diversidade que a família Fabaceae apresenta no Brasil, com aproximadamente 1500 espécies e 175 gêneros (Souza e Lorenzi 2008). Outras famílias de hospedeiras que se destacaram nesse e em outros estudos foram Lauraceae (quinta mais diversa em Julião et al. 2005), Chrysobalanaceae (segunda mais diversa em Almada \& Fernandes 2011), Sapotaceae (quarta mais diversa em Julião et al. 2005) e Burseraceae (família mais diversa no levantamento de Maia 2011) 
Tabela 2. Distribuição do número de morfotipos de galhas e de espécies de plantas entre as 10 famílias mais representativas na Floresta Ombrófila Densa em Porto Trombetas, Pará, Brasil.

\begin{tabular}{lcc}
\hline Família & Número de morfotipos de galhas & Número de espécies de plantas \\
\hline Fabaceae & 18 & 9 \\
Bignoniaceae & 14 & 3 \\
Sapotaceae & 12 & 5 \\
Lauraceae & 12 & 5 \\
Burseraceae & 10 & 4 \\
Meliaceae & 4 & 2 \\
Chrysobalanaceae & 4 & 4 \\
Violaceae & 3 & 2 \\
Sapindaceae & 3 & 3 \\
Piperaceae & 3 & 2 \\
\hline Outras famílias & 29 & 26 \\
\hline
\end{tabular}

Os gêneros Adenocalymma (Bignoniaceae), Pouteria (Sapotaceae), Inga (Fabaceae) e Protium (Burseraceae) foram os mais diversos em número de morfotipos de galhas $(13,12,11$ e sete, respectivamente). Previamente, apenas quatro morfotipos foram registrados no gênero Adenocalymma (Memora) na Floresta Nacional SaracáTaquera (Almada \& Fernandes 2011). Para o gênero Protium (15 spp.) foram registradas 98 espécies de insetos galhadores na Reserva Florestal Ducke em Manaus, AM (Fernandes 2010). Esse gênero também foi o mais diverso no levantamento de galhas do Platô Bacaba com 17 morfotipos (Maia 2011). A espécie Adenocalymma neoflavidum (Bignoniaceae) foi a planta hospedeira com o maior número de morfotipos de galhas, com nove espécies. Outras espécies que merecem destaque são Ocotea sp. (Lauraceae), Inga sp. e Pouteria sp., que hospedaram seis, cinco e cinco morfotipos de galhas, respectivamente.

O órgão de ocorrência de galhas mais frequente foi a superfície das folhas $(72,3 \%)$. Galhas na nervura central $(16,1 \%)$ e no caule $(7,1 \%)$ também foram relativamente comuns. Menos frequente foi a ocorrência de galhas na gema apical $(2,7 \%)$ e gavinha $(1,8 \%)$. Segundo Fernandes et al. (1988) a grande maioria das galhas tropicais ocorrem nas folhas. Resultados similares também foram observados por Almada \& Fernandes (2011) e Maia (2011), onde $89 \%$ e $80 \%$ das galhas observadas ocorreram nas folhas, respectivamente.

As galhas registradas apresentaram ampla variedade de formas e colorações (Tabela 3). A forma de galha mais comum foi discóide (35,7\%), seguida de globóide $(28,6 \%)$ e elipsóide $(23,2 \%)$. Outras formas ocorrentes foram amorfa $(8,9 \%)$ e coniforme $(3,6 \%)$. Quanto à coloração, galhas marrons foram as mais frequentes 
$(67,9 \%)$, seguidas por galhas verdes $(24,1 \%)$ e amarelas $(5,4 \%)$. Galhas beges, brancas e vermelhas somaram 2,6\% (Tabela 3). Quanto à superfície, galhas glabras representaram $86,6 \%$ do total contra apenas $13,4 \%$ das galhas pilosas. Morfologia semelhante foi observada no levantamento de Almada\& Fernandes (2011) onde os morfotipos mais comuns foram de galhas discóides (48\%), verdes (67\%) e glabras $(96 \%)$.

Tabela 3. Órgão de ocorrência, forma e coloração dos morfotipos de galhas ocorrentes em Floresta Ombrófila Densa em Porto Trombetas, Pará, Brasil.

\begin{tabular}{lcclccccc}
\hline Órgão & N. & $\%$ & \multicolumn{2}{c}{ N. } & \multicolumn{2}{c}{ N. } \\
Forfotipos & $\%$ & Forma & morfotipos & $\%$ & Cor & morfotipos & $\%$ \\
Nervura central & 18 & 72,3 & Discóide & 40 & 35,7 & Marrom & 76 & 67,9 \\
Caule & 8 & 7,1 & Elipsóide & 26 & 23,2 & Amarela & 6 & 5,4 \\
Gema apical & 3 & 2,7 & Amorfa & 10 & 8,9 & Vermelha & 1 & 0,9 \\
Gavinha & 2 & 1,8 & Coniforme & 4 & 3,6 & Branca/Beje & 2 & 1,8 \\
\hline
\end{tabular}

O número de morfotipos de galhas observados nesse estudo (112) é intermediário quando comparado aos outros estudos de diversidade de galhas na Amazônia. Yukawa et al. (2001), por exemplo, fizeram um levantamento de artrópodes galhadores em áreas úmidas de Manaus (AM) registrando 109 espécies. Em outro estudo, Julião et al. (2005) registraram 236 morfotipos de galhas no dossel de áreas de várzea em Mamirauá (AM). No estudo de Almada \& Fernandes (2011), das 309 espécies de galhadores registradas, apenas 167 ocorreram em áreas de floresta primária, número um pouco maior ao observado no presente estudo. A riqueza de galhas no local de estudo também foi elevada diante dos levantamentos realizados no Cerrado (por exemplo, Coelho et al. 2009; Araújo et al. 2011; com 92 e 62 morfotipos de galhas, respectivamente), com exceção de Carneiro et al. (2009b) que registraram 241 espécies de galhadores na Cadeia do Espinhaço, MG.

$\mathrm{O}$ crescente número de estudos realizados na Amazônia brasileira tem mostrado o potencial da região para a diversidade de insetos galhadores. Alguns desses estudos tem evidenciado que as galhas podem ser boas ferramentas para o monitoramento da saúde da floresta (Fernandes et al. 2010) e também para a aferição dos impactos causados pelas atividades humanas (Araújo \& Espírito-Santo Filho 2012). Desse modo, futuros inventários de galhadores e plantas hospedeiras, permitirão grandes avanços no conhecimento dessa guilda na Amazônia, principalmente, pela alta diversidade e a grande área geográfica que a floresta representa no Brasil e na América do Sul. 
Tabela 1. Ocorrência e caracterização de galhas de insetos em plantas hospedeiras em sub-bosque de Floresta Ombrófila Densa em Porto Trombetas, Pará, Brasil.

\begin{tabular}{|c|c|c|c|c|c|c|c|}
\hline Família & Espécie & Galhador & Órgão & Forma & Cor & Pubescência & $\begin{array}{l}\text { Tamanho } \\
\text { (cm) }\end{array}$ \\
\hline Annonaceae & Duguetia sp. & Indeterminado & Folha & Globóide & Marrom & Pilosa & 0,3 \\
\hline \multirow[t]{3}{*}{ Apocynaceae } & Bonafousia sp. & Indeterminado & Folha & Amorfa & Marrom & Glabra & 0,5 \\
\hline & $\begin{array}{l}\text { Geissospermum urceolatum A.H. } \\
\text { Gentry }\end{array}$ & Indeterminado & Folha & Discóide & Marrom & Glabra & 0,5 \\
\hline & Tabernaemontana catharinensis A.DC. & Indeterminado & Folha & Globóide & Marrom & Glabra & 0,2 \\
\hline \multirow[t]{12}{*}{ Bignoniaceae } & $\begin{array}{l}\text { Adenocalymma neoflavidum } \\
\text { L.G.Lohmann }\end{array}$ & Indeterminado & Caule & Elipsóide & Marrom & Glabra & $2,0 / 1,0$ \\
\hline & & Indeterminado & Caule & Elipsóide & Verde & Glabra & $0,4 / 0,1$ \\
\hline & & Indeterminado & Folha & Discóide & Marrom & Glabra & 0,5 \\
\hline & & Indeterminado & Folha & Discóide & Verde & Glabra & 0,3 \\
\hline & & Indeterminado & Folha & Elipsóide & Marrom & Glabra & $0,5 / 0,3$ \\
\hline & & Indeterminado & Folha & Globóide & Beje & Glabra & 0,3 \\
\hline & & Indeterminado & Folha & Globóide & Vermelha & Pilosa & 0,3 \\
\hline & & Cecidomyiidae & $\begin{array}{l}\text { Gavinha e } \\
\text { nervura central }\end{array}$ & Globóide & Verde & Glabra & 0,5 \\
\hline & & Cecidomyiidae & Nervura central & Elipsóide & Verde & Glabra & $0,6 / 0,3$ \\
\hline & Adenocalymma sp. & Indeterminado & Folha & Amorfa & Verde & Glabra & 0,3 \\
\hline & & Indeterminado & Folha & Discóide & Verde & Glabra & 0,2 \\
\hline & & Cecidomyiidae & $\begin{array}{l}\text { Gavinha e } \\
\text { nervura central }\end{array}$ & Elipsóide & Verde & Glabra & $1 / 0,5$ \\
\hline
\end{tabular}


Tabela 1. Contin.

\begin{tabular}{|c|c|c|c|c|c|c|c|}
\hline & & Indeterminado & Gema apical & Amorfa & Verde & Glabra & 0,8 \\
\hline & $\begin{array}{l}\text { Amphilophium pulverulentum } \\
\text { (Sandwith) L.G.Lohmann }\end{array}$ & Indeterminado & Folha & Elipsóide & Amarela & Glabra & $0,8 / 0,4$ \\
\hline Bombacaceae & $\begin{array}{c}\text { Quararibea ochrocalyx (K.Schum.) } \\
\text { Vischer }\end{array}$ & Indeterminado & Folha & Discóide & Verde & Glabra & 0,1 \\
\hline Boraginaceae & Cordia sp. & Indeterminado & Folha & Globóide com & Marrom & Glabra & $0,4 / 0,3$ \\
\hline \multirow[t]{10}{*}{ Burseraceae } & Dacryodes microcarpa Cuatrec. & Indeterminado & Folha & Coniforme & Verde & Glabra & $0,5 / 0,3$ \\
\hline & & Indeterminado & Folha & Discóide & Amarela & Glabra & 0,4 \\
\hline & & Indeterminado & Nervura central & Elipsóide & Marrom & Glabra & $0,6 / 0,3$ \\
\hline & Protium apiculatum Swart & Indeterminado & Caule & Elipsóide & Marrom & Pilosa & $0,5 / 0,2$ \\
\hline & & Indeterminado & Folha & Discóide & Verde & Glabra & 0,2 \\
\hline & Protium aracouchini Marchand & Indeterminado & Folha & Globóide & Marrom & Glabra & 0,2 \\
\hline & Protium sp. & Indeterminado & Folha & Discóide & Marrom & Glabra & 0,2 \\
\hline & & Indeterminado & Folha & Discóide & Marrom & Glabra & 0,2 \\
\hline & & Indeterminado & Nervura central & Globóide & Marrom & Glabra & 0,3 \\
\hline & & Indeterminado & Nervura central & Globóide & Verde & Glabra & 0,4 \\
\hline Cecropiaceae & Pourouma sp. & Cecidomyiidae & Folha & Discóide & Verde & Glabra & 0,3 \\
\hline \multirow[t]{2}{*}{ Chrysobalanaceae } & Hirtella bicornis Mart. \& Zucc. & Indeterminado & Folha & Globóide & Marrom & Pilosa & 0,5 \\
\hline & $\begin{array}{l}\text { Licania pallida (Hook.f.) Spruce ex } \\
\text { Sagot }\end{array}$ & Cecidomyiidae & Folha & Discóide & Amarela & Glabra & 0,1 \\
\hline \multirow[t]{2}{*}{ Chrysobalanaceae } & Licania sp. & Indeterminado & Folha & Discóide & Verde & Glabra & 0,3 \\
\hline & Licania sprucei (Hook.f.) Fritsch. & Indeterminado & Folha & Discóide & Verde & Glabra & 0,3 \\
\hline
\end{tabular}


Tabela 1. Contin

\begin{tabular}{|c|c|c|c|c|c|c|c|}
\hline Clusiaceae & Clusia sp. & Indeterminado & Nervura central & Elipsóide & Verde & Glabra & $0,8 / 0,4$ \\
\hline Dichapetalaceae & Tapura guianensis Aubl. & Indeterminado & Nervura central & Globóide & Marrom & Glabra & 0,4 \\
\hline Elaeocarpaceae & Sloanea grandiflora $\mathrm{Sm}$. & Indeterminado & Folha & Discóide & Marrom & Glabra & 0,4 \\
\hline Euphorbiaceae & Pausandra macropetala Ducke & Indeterminado & Folha & Globóide & Verde & Glabra & \\
\hline \multirow[t]{16}{*}{ Fabaceae } & Bauhinia sp. & Indeterminado & Folha & Elipsóide & Marrom & Glabra & $0,6 / 0,1$ \\
\hline & & Indeterminado & Nervura central & Elipsóide & Verde & Pilosa & $1 / 0,3$ \\
\hline & Bocoa viridiflora (Ducke) Cowan & Indeterminado & Folha & Discóide & Amarela & Glabra & 0,2 \\
\hline & & Indeterminado & Nervura central & Globóide & Marrom & Glabra & 0,3 \\
\hline & Hymenaea courbaril L. & Cecidomyiidae & Folha & Discóide & Verde & Glabra & 0,2 \\
\hline & Hymenaea sp. & Indeterminado & Folha & Discóide & Marrom & Glabra & 0,3 \\
\hline & Inga alba (Sw.) Willd. & Cecidomyiidae & Folha & Discóide & Marrom & Glabra & 0,5 \\
\hline & & Indeterminado & Nervura central & Elipsóide & Marrom & Glabra & $0,2 / 0,1$ \\
\hline & Inga brachystachys (Ducke) Ducke & Indeterminado & Folha & Globóide & Marrom & Glabra & 0,3 \\
\hline & & Indeterminado & Nervura central & Elipsóide & Marrom & Glabra & $0,8 / 0,4$ \\
\hline & Inga sp. & Indeterminado & Caule & Globóide & Marrom & Glabra & 0,6 \\
\hline & & Indeterminado & Folha & Discóide & Amarela & Glabra & 0,2 \\
\hline & & Indeterminado & Folha & Discóide & Marrom & Glabra & 0,2 \\
\hline & & Indeterminado & Folha & Globóide & Marrom & Pilosa & 0,4 \\
\hline & & Indeterminado & Nervura central & Elipsóide & Marrom & Glabra & $0,9 / 0,2$ \\
\hline & Inga stipularis DC. & Indeterminado & Folha & Globóide & Marrom & Pilosa & 0,4 \\
\hline
\end{tabular}


Tabela 1. Contin

\begin{tabular}{|c|c|c|c|c|c|c|c|}
\hline & & Indeterminado & Nervura central & Elipsóide & Verde & Glabra & $1 / 0,3$ \\
\hline & Peltogyne venosa (M.Vahl) Benth. & Indeterminado & Folha & Discóide & Marrom & Glabra & 0,2 \\
\hline \multirow[t]{2}{*}{ Flacourtiaceae } & Casearia javitensis Kunth & Indeterminado & Nervura central & Elipsóide & Marrom & Glabra & $0,6 / 0,3$ \\
\hline & Casearia sp. & Indeterminado & Folha & Discóide & Marrom & Glabra & 0,2 \\
\hline Hugoniaceae & Roucheria sp. & Indeterminado & Nervura central & Amorfa & Marrom & Glabra & 0,1 \\
\hline \multirow[t]{2}{*}{ Lacistemataceae } & Lacistema grandiflora Schnizl. & Indeterminado & Folha & Discóide & Marrom & Glabra & 0,2 \\
\hline & & Cecidomyiidae & Folha & Globóide & Verde & Glabra & 0,8 \\
\hline \multirow[t]{12}{*}{ Lauraceae } & Aniba canellila (Kunth) Mez & Indeterminado & Caule & Amorfa & Marrom & Glabra & 0,2 \\
\hline & & Indeterminado & Folha & Discóide & Marrom & Glabra & 0,2 \\
\hline & Aniba ferrea Kubitzki & Indeterminado & Folha & Elipsóide & Marrom & Glabra & $0,8 / 0,4$ \\
\hline & & Cecidomyiidae & Folha & Globóide & Marrom & Pilosa & 0,5 \\
\hline & Anibia sp. & Indeterminado & Folha & Discóide & Marrom & Glabra & 0,2 \\
\hline & Licaria sp. & Indeterminado & Folha & Discóide & Marrom & Glabra & 0,2 \\
\hline & Ocotea sp. & Indeterminado & Folha & Amorfa & Marrom & Pilosa & 0,5 \\
\hline & & Indeterminado & Folha & Coniforme & Marrom & Glabra & 0,4 \\
\hline & & Indeterminado & Folha & Discóide & Marrom & Glabra & 0,2 \\
\hline & & Indeterminado & Folha & Discóide & Marrom & Glabra & 0,3 \\
\hline & & Indeterminado & Folha & Globóide & Branca & Pilosa & 0,3 \\
\hline & & Indeterminado & Folha & Globóide & Marrom & Glabra & 0,5 \\
\hline Melastomataceae & Miconia sp. & Cecidomyiidae & Folha & Globóide & Marrom & Glabra & 0,4 \\
\hline
\end{tabular}


Tabela 1. Contin

\begin{tabular}{|c|c|c|c|c|c|c|c|}
\hline & & Indeterminado & Folha & Globóide & Marrom & Pilosa & 0,8 \\
\hline & & Indeterminado & Nervura central & Elipsóide & Verde & Pilosa & $1,5 / 0,8$ \\
\hline \multirow[t]{4}{*}{ Meliaceae } & $\begin{array}{l}\text { Trichilia appendiculata (Triana \& } \\
\text { Planch.) C. DC. }\end{array}$ & Indeterminado & Folha & Globóide & Marrom & Glabra & 0,3 \\
\hline & Trichilia sp. & Indeterminado & Caule & Elipsóide & Marrom & Glabra & $0,8 / 0,3$ \\
\hline & & Indeterminado & Folha & Coniforme & Marrom & Pilosa & $0,6 / 0,2$ \\
\hline & & Indeterminado & Folha & Elipsóide & Marrom & Glabra & $0,5 / 0,3$ \\
\hline Monimiaceae & Siparuna guianensis Aubl. & Cecidomyiidae & Folha & Globóide & Marrom & Glabra & 0,3 \\
\hline Moraceae & Sorocea guilleminiana Gaudich. & Indeterminado & Folha & Amorfa & Marrom & Glabra & 0,5 \\
\hline Myrsinaceae & Cybianthus guyanensis (A.DC.) Miq. & Indeterminado & Nervura central & Globóide & Marrom & Glabra & 0,4 \\
\hline Myrtaceae & Myrcia sp. & Indeterminado & Folha & Discóide & Verde & Glabra & 0,4 \\
\hline Ochnaceae & Ouratea sp. & Indeterminado & Folha & Discóide & Verde & Glabra & 0,2 \\
\hline \multirow[t]{3}{*}{ Piperaceae } & Piper bartlingianum (Miq.) C.DC. & Indeterminado & Folha & Discóide & Marrom & Glabra & 0,2 \\
\hline & & Indeterminado & Gema apical & Amorfa & Verde & Glabra & 1 \\
\hline & Piper sp. & Indeterminado & Folha & Discóide & Marrom & Glabra & 0,5 \\
\hline Polygalaceae & Moutabea guianensis Aubl. & Indeterminado & Nervura central & Amorfa & Marrom & Glabra & 0,3 \\
\hline Polygonaceae & Symmeria sp. & Indeterminado & Folha & Elipsóide & Marrom & Glabra & $0,5 / 0,4$ \\
\hline Rubiaceae & Palicourea virens (Poepp.) Standl. & Indeterminado & Folha & Discóide & Amarela & Glabra & 0,3 \\
\hline \multirow[t]{3}{*}{ Sapindaceae } & Matayba sp. & Indeterminado & Folha & Discóide & Marrom & Glabra & 0,3 \\
\hline & Paullinia pachycarpa Benth. & Indeterminado & Folha & Discóide & Marrom & Glabra & 0,3 \\
\hline & Serjania sp. & Indeterminado & Folha & Discóide & Marrom & Glabra & 0,3 \\
\hline
\end{tabular}


Tabela 1. Contin

\begin{tabular}{|c|c|c|c|c|c|c|c|}
\hline \multirow[t]{12}{*}{ Sapotaceae } & Pouteria erythrochrysa T.D.Penn. & Cecidomyiidae & Folha & Coniforme & Marrom & Glabra & $0,1 / 0,05$ \\
\hline & & Indeterminado & Folha & Discóide & Marrom & Glabra & 0,3 \\
\hline & & Indeterminado & Gema apical & Amorfa & Marrom & Glabra & 0,5 \\
\hline & & Indeterminado & Nervura central & Elipsóide & Marrom & Glabra & $0,9 / 0,3$ \\
\hline & Pouteria guianensis Aubl. & Indeterminado & Folha & Discóide & Marrom & Glabra & 0,5 \\
\hline & Pouteria macrocarpa (Mart.) D.Dietr. & Indeterminado & Folha & Globóide & Marrom & Pilosa & $0,8 / 0,3$ \\
\hline & Pouteria sp. & Indeterminado & Caule & Globóide & Marrom & Glabra & 0,8 \\
\hline & & Indeterminado & Folha & Discóide & Marrom & Glabra & 0,3 \\
\hline & & Indeterminado & Folha & Elipsóide & Verde & Glabra & $0,8 / 0,4$ \\
\hline & & Indeterminado & Folha & Elipsóide & Verde & Glabra & $0,8 / 0,4$ \\
\hline & & Indeterminado & Folha & Globóide & Marrom & Pilosa & 0,3 \\
\hline & Pouteria vernicosa T.D.Penn. & Indeterminado & Folha & Globóide & Marrom & Glabra & 0,3 \\
\hline Simaroubaceae & Simarouba sp. & Indeterminado & Folha & Globóide & Marrom & Glabra & 0,3 \\
\hline Sterculiaceae & Theobroma sp. & Indeterminado & Folha & Elipsóide & Marrom & Glabra & 0,4 \\
\hline \multirow[t]{3}{*}{ Violaceae } & Paypayrola grandiflora Tul. & Indeterminado & Folha & Globóide & Marrom & Glabra & 0,2 \\
\hline & $\begin{array}{l}\text { Rinorea macrocarpa (Mart. ex Eichler) } \\
\text { Kuntze }\end{array}$ & Indeterminado & Caule & Elipsóide & Marrom & Glabra & $0,8 / 0,4$ \\
\hline & & Indeterminado & Folha & Discóide & Marrom & Glabra & 0,4 \\
\hline
\end{tabular}




\section{Agradecimentos}

Agradecemos à Genimar Rebouças Julião pelo auxílio na identificação das plantas; Ao Guilherme Ferreira e Sr. Colé pelo auxílio nos trabalhos de campo; ao ICMBio Porto Trombetas pelo licenciamento; à Mineração Rio Norte - MRN pelo financiamento e oportunidade de desenvolvimento do projeto; à STCP pelo apoio logístico e suporte.

\section{Referências}

Almada, E.D.; Fernandes, G.W. 2011. Insetos indutores de galhas em florestas de terra firme e em reflorestamentos com espécies nativas na Amazônia Oriental, Pará, Brasil. Boletim do Museu Paraense Emilio Goeldi 6: 163-196.

Araújo, W.S.; Espírito-Santo Filho, K. 2012. Edge effect benefits galling insects in the Brazilian Amazon. Biodiversity and Conservation 21: 2991-2997.

Araújo, W.S.; Santos, B.B.; Gomes-Klein, V.L. 2011. Insect galls from Serra dos Pireneus, GO, Brazil. Biota Neotropica 11: 357-365.

Carneiro, M.A.A.;Branco, C.S.A.; Braga, C.E.D.;Almada, E.D.; Costa, M.B.M.; Maia, V.C.; Fernandes, G.W. 2009a Are gall midge species (Diptera, Cecidomyiidae) host-plant specialists? Revista Brasileira de Entomologia 53: 365-378.

Carneiro, M.A.A.; Borges, R.A.X.; Araújo, A.P.A.; Fernandes, G.W. 2009b. Insetos indutores de galhas da porção sul da Cadeia do Espinhaço, Minas Gerais, Brasil. Revista Brasileira de Entomologia 53: 570-592.

Coelho, M.S.; Almada, E.D.; Fernandes, G.W.; Carneiro, M.A.A.; Santos, R.M.; Quintino, A.V.; Sanchez-Azofeifa, A. 2009. Gall inducing arthropods from a seasonally dry tropical Forest in Serra do Cipó, Brazil. Revista Brasileira de Entomologia 53: 404-414.

Fernandes, G.W.A.;Tameirão-Neto, E.;Martins, R.G.1988.Ocorrência e caracterização de galhas entomógenas na vegetação do Campus Pampulha da Universidade Federal de Minas Gerais. Revista Brasileira de Zoologia 5: 11-29.

Fernandes, S.P.C.; Castelo-Branco, B.P.; Albuquerque, F.A.; Ferreira, A.L.N.; BritoRamos, A.B.; Braga, D.V.V.; Almeida-Cortez, J. 2009. Galhas entomógenas em um fragmento urbano de Mata Atlântica no centro de endemismo de Pernambuco. Revista Brasileira de Biociências 7: 240-244.

Fernandes, S.P.C. 2010. Insetos galhadores associados à família Burseraceae da

Reserva Florestal Ducke, Manaus-AM. Dissertação de Mestrado, Instituto Nacional de Pesquisas da Amazônia, Manaus.

Fernandes, G.W.;Almada, E.D.; Carneiro, M.A.A. 2010. Gall-inducing insect richness as indicators of forest age and health. Environmental Entomology 39: 11341140 .

Godfray, H.C.J.; Lewis, O.T.; Memmott, J. 1999. Studying insect diversity in the tropics. Philosophical Transactions of the Royal Society B 354: 1811-1824. 
IBGE - Instituto Brasileiro de Geografia e Estatística. 1992. Manual técnico da vegetação brasileira. Rio de Janeiro: IBGE. (Séries manuais Técnicos de Geociências).

Julião, G.R.; Venticinque, E.M.; Fernandes, G.W. 2005. Richness and abundance of gall-forming insects in the Mamirauá Várzea, a flooded Amazonian forest. Uakari 1: 39-42.

Lewinsohn, T.M.; Novotny, V.; Basset, Y. 2005. Insects on plants: diversity of herbivore assemblages revisited. Annual Review of Ecology Evolution and Systematic 36: 597-620.

Maia, V.C. 2001. The gall midges (Diptera, Cecidomyiidae) from three restingas of Rio de Janeiro State, Brazil. Revista Brasileira de Zoologia 18: 583-629.

Maia, V.C. 2011. Characterization of insect galls, gall makers, and associated fauna of Platô Bacaba (Porto de Trombetas, Pará, Brazil). Biota Neotropica 11: 37-53.

Maia, V.C.; Fernandes, G.W. 2004. Insect galls from Serra de São José (Tiradentes, MG, Brazil). Brazilian Journal of Biology 64: 423-445.

Maia, V. C.; Magenta, M. A. G.; Martins, S. E. 2008. Occurrence and characterization of insect galls at restinga áreas of Bertioga (São Paulo, Brazil). Biota Neotropica 8: 167-197.

Santos, B.B.; Ferreira, H.D.; Araújo, W.S. 2010. Ocorrência e caracterização de galhas entomógenas em uma área de floresta estacional semidecídua em Goiânia, Goiás, Brasil. Acta Botanica Brasilica 24: 243-249.

Souza, V.C.; H. Lorenzi. 2008. Botânica sistemática: guia ilustrado para identificação das famílias de Fanerógamas nativas e exóticas no Brasil, baseado em APG II. $2^{\circ}$ ed. Nova Odessa, SP: Instituto Plantarum. 2 Ed. São Paulo: Nova Odessa.

Stone, G.N.; Schönrogge, K.. 2003. The adaptive significance of insect gall morphology. Trends in Ecology and Evolution 18: 512-522.

The Plant List. 2010. The Plant List. Version 1. http://www.theplantlist.org/. Acesso em 27 jun. 2012.

Yukawa, J.; Tokuda, M.; Uechi, N.; Sato, S. 2001. Species richness of galling arthropods in Manaus, Amazon and the surroundings of the Iguassu Falls. Ekasia 41: 11-15. 